\title{
Regularity estimates via the entropy dissipation for the spatially homogeneous Boltzmann equation without cut-off
}

Cédric Villani

Abstract. We show that in the setting of the spatially homogeneous Boltzmann equation without cut-off, the entropy dissipation associated to a function $f \in L^{1}\left(\mathbb{R}^{N}\right)$ yields a control of $\sqrt{f}$ in Sobolev norms as soon as $f$ is locally bounded below. Under this additional assumption of lower bound, our result is an improvement of a recent estimate given by P.-L. Lions, and is optimal in a certain sense.

\section{Introduction.}

The Boltzmann equation in the kinetic theory of gases is one of the fundamental models for nonequilibrium statistical mechanics. The gas is modelled by a density function $f(t, x, v) \geq 0$ on the extended phase space of particles, such that

$$
\frac{\partial f}{\partial t}+v \cdot \nabla_{x} f=Q(f, f), \quad t \geq 0, x \in \mathbb{R}^{N}, v \in \mathbb{R}^{N}
$$


where $Q(f, f)$ is the Boltzmann collision operator, which acts only on the velocity variable $v$. If $f$ is a function of $v \in \mathbb{R}^{N}$, it is defined by

$$
Q(f, f)=\int_{\mathbb{R}^{N}} d v_{*} \int_{S^{N-1}} d \omega B\left(v-v_{*}, \omega\right)\left(f^{\prime} f_{*}^{\prime}-f f_{*}\right),
$$

where $S^{N-1}$ is the unit sphere in $\mathbb{R}^{N}, f^{\prime}=f\left(v^{\prime}\right)$, and so on, and

$$
\left\{\begin{array}{l}
v^{\prime}=v-\left(v-v_{*}, \omega\right) \omega, \\
v_{*}^{\prime}=v_{*}+\left(v-v_{*}, \omega\right) \omega .
\end{array}\right.
$$

The kernel, or cross-section, $B: \mathbb{R}^{N} \times S^{N-1} \longrightarrow \mathbb{R}_{+}$is a weight function modelling the interaction, such that $B(z, \omega)$ depends only on $|z|$ and $(z /|z|, \omega)$.

The great majority of mathematical works upon the Boltzmann equation is based on the assumption that $B$ is locally integrable on $\mathbb{R}^{N} \times S^{N-1}$. However, this assumption is often unsatisfactory from the physical point of view, since it is always false if the particles interact through forces of infinite range [20], [3], [24]. In particular, for inverse power laws, $B(z, \omega)=|z|^{\gamma} b(\cos \alpha)$ with $\cos \alpha=(z /|z|, \omega), \gamma=(s-$ $(2 N-1)) /(s-1)$, and if $N=3, b$ has a singularity of order $(s+1) /(s-1)$ as $\cos \alpha \longrightarrow 0$. In this work, we shall precisely focus on the case where $B$ is singular.

We shall only be concerned with the spatially homogeneous case, i.e. when the unknown in (1) is assumed not to depend on $x$, so that (1) simply reads

$$
\frac{\partial f}{\partial t}=Q(f, f), \quad t \geq 0, v \in \mathbb{R}^{N} .
$$

(we refer to [19] for partial results in the inhomogeneous case). For this equation, there is by now a fairly complete theory of existence in an $L^{1}$ setting for non cut-off potentials, which covers all the physically interesting potentials [3], [15], [24]. In the last reference, we also showed how one could rigorously derive the (spatially homogeneous) Landau equation for plasmas, which is the equation corresponding to (4) in the case of Coulomb interactions.

Apart from existence results, very little is known from the analytical point of view. However, it is conjectured that, due to the nonintegrable singularity in $B$, solutions to (4) become smooth for positive times (which is false for cut-off kernels). The likelihood of this conjecture is reinforced by the study of the Landau equation [18], [4], [13], 
which is obtained from the Boltzmann equation by "concentrating on grazing collisions", and has definite smoothing (and compactifying) effects. In particular, (in the homogeneous case) its solutions become $C^{\infty}$ for positive times, at least for the so-called "hard potentials" (see [13] for precise statements and complete proofs).

The smoothing conjecture for the Boltzmann equation was tackled by Desvillettes [10], [11], [12] and Proutière [21], in rather particular cases, with the help of Fourier representations. The proofs are however very technical, and depend highly upon the dimension $N$. In the aforementioned works, the case of radially symmetric data in 2 dimensions is treated (or non radially symmetric if $\gamma=0$; for more complicated cases, the proofs have still not been written down. Moreover, some unnatural smoothing of the kinetic cross-section is needed (while $|z|^{\gamma}, 0<\gamma<1$, is not smooth near $z=0$ ).

In [13], a different strategy was followed for proving smoothness in the Landau equation. The proof is at the same time simpler and independent on the dimension. Our aim here is to give a (loosely related) possible startpoint for a complete study of regularization effects in the Boltzmann equation, by showing that the usual estimate on the entropy dissipation automatically entails such an effect.

More precisely, let us define

(5) $\bar{D}(f) \equiv \int_{\mathbb{R}^{2 N}} d v d v_{*} \int_{S^{N-1}} d \omega B\left(v-v_{*}, \omega\right)\left(\sqrt{f^{\prime} f_{*}^{\prime}}-\sqrt{f f_{*}}\right)^{2} \geq 0$.

The functional $\bar{D}$ is well-defined (possibly infinite) on $L^{1}\left(\mathbb{R}^{N}\right)$, for instance by the use of the joint convexity of $(x, y) \longrightarrow(\sqrt{x}-\sqrt{y})^{2}$ on $\mathbb{R}^{2}$. It is clear that $\bar{D}$ is related to the usual entropy dissipation functional,

(6) $D(f) \equiv \frac{1}{4} \int_{\mathbb{R}^{2 N}} d v d v_{*} \int_{S^{N-1}} d \omega B\left(v-v_{*}, \omega\right)\left(f^{\prime} f_{*}^{\prime}-f f_{*}\right) \log \frac{f^{\prime} f_{*}^{\prime}}{f f_{*}}$

since, by the classical inequality $(x-y) \log (x / y) \geq 4(\sqrt{x}-\sqrt{y})^{2}$, one has

$$
D(f) \geq \bar{D}(f) .
$$

Our main result is essentially the following. Let $f \in L_{+}^{1}\left(\mathbb{R}^{N}\right)$, such that $\bar{D}(f)$ is finite, and assume that

$$
B(z, \omega) \geq \Phi(|z|) b(\cos \alpha)
$$


where $\Phi$ is smooth and bounded below away from 0 and infinity, and $b$ has a singularity of order $1+\nu, \nu>0$. Then, if $f$ is locally bounded from below,

$$
\sqrt{f} \in H_{\mathrm{loc}}^{\nu / 2} .
$$

As an immediate consequence of this estimate, solutions of (4) for an initial datum which has finite entropy, and is locally bounded below, will satisfy for all $R, T>0$

$$
\int_{0}^{T} d t\|\sqrt{f}(t, \cdot)\|_{H^{\nu / 2}(|v|<R)}^{2}<\infty,
$$

since a lower bound is known to exist for these solutions [8] (see also [22] in the cut-off case).

Closely related results have been obtained recently by Lions [19]. Before we comment on them, it may be of interest to briefly track the idea that smoothness estimates for the Boltzmann equation should be obtained naturally for $\sqrt{f}$ instead of $f$. First of all, such estimates have been sought for a long time in the context of Maxwellian potentials (when $B$ depends only on $(z /|z|, \omega)$ ). Indeed, it is now known that in this case, the Fisher information

$$
I(f)=4\|\sqrt{f}\|_{\dot{H}^{1}}^{2}=4 \int_{\mathbb{R}^{N}}|\nabla \sqrt{f}|^{2}
$$

is a Lyapunov functional [16], [23], [7], [25]. Complete proofs are given in the last reference.

In a more general setting, regularity estimates for $\sqrt{f}$ and entropy dissipation estimates are associated together in works by Lions [17], [19] and the author [24] (see also Cercignani [9]). In [24], it is shown that $\bar{D}(f)$ yields sufficient regularity on the tensor product $\sqrt{f f_{*}}$ to give a meaning to (4) even for very singular and very soft potentials (i.e. $\gamma<-2)$. More generally, $D(f)$ gives some control on the regularity of $\sqrt{f f_{*}}$. It is then a natural question whether this estimate for $\sqrt{f}$ implies a control of $f$ itself. A first strategy to answer this problem is to introduce a well-chosen artificial weight-function $\hat{B}\left(v-v_{*}, \omega\right)$, and integrate the estimate for $\sqrt{f^{\prime} f_{*}^{\prime}}-\sqrt{f f_{*}}$ in $v_{*}$ and $\omega$ after multiplication by $\hat{B}$, thus obtaining an estimate on

$$
\begin{aligned}
\hat{Q}(\sqrt{f}, \sqrt{f}) & =\int d v_{*} d \omega \hat{B}\left(v-v_{*}, \omega\right)\left(\sqrt{f^{\prime} f_{*}^{\prime}}-\sqrt{f f_{*}}\right) \\
& \equiv \hat{Q}^{+}(\sqrt{f}, \sqrt{f})-\hat{Q}^{-}(\sqrt{f}, \sqrt{f}) .
\end{aligned}
$$


Then one uses the regularity properties of $Q^{+}$in $L^{2}$ (cf. [17], [26], [5]), which is of course the natural space for $\sqrt{f}$, and the simple form of $Q^{-}$. This is what Lions does in [17] (to characterize equilibria distributions for (1) under very little assumptions) and in [19], to prove an estimate of the same kind as (8), namely

$$
\sqrt{f} \in H_{\mathrm{loc}}^{s}, \quad \text { for all } s<s_{1}=\frac{\nu}{2}\left(\frac{1}{1+\frac{\nu}{N-1}}\right) .
$$

The exponent is hence not so good as the one in (8), but a lower bound is not needed for it. The proof by Lions is very simple, but relies on the deep result of smoothing of the positive part of Boltzmann's collision operator. It is possible that a better knowledge of the explicit constants in this result (see [5] for some of them) could lead to (8). In any case, our proof implies that $\nu / 2$ is the optimal exponent, in the sense that for all $\varepsilon>0$ one can find a function $f$ such that

$$
\bar{D}(f)<\infty \quad \text { and } \quad\|\sqrt{f}\|_{\dot{H}^{\nu / 2+\varepsilon}}^{2}=\infty .
$$

Moreover, our proof is elementary and relies only upon careful changes of variables. As far as the physical meaning is concerned, it is another illustration of the general principle that the entropy dissipation yields regularity "along the collisions", either in the tensor phase space $\mathbb{R}^{2 N}$ (via estimates on $\sqrt{f^{\prime} f_{*}^{\prime}}-\sqrt{f f_{*}}$ ), or in $\mathbb{R}^{N}$ (via estimates on $\sqrt{f^{\prime}}-\sqrt{f}$ ). See [14] for still another manifestation of this principle.

The only drawback of this method is the need for a lower bound. It is possible that our computation can be refined in such a way to dispend with this assumption, maybe at the loss of the optimal exponent $\nu / 2$. In the end of the paper, we give possible hints for this. However, we shall not go further, since on one hand Lions's result is general enough to cover all the cases when one is not interested in the exact exponent (in particular for compactness properties associated to the complete equation (1)), and on the other hand a pointwise lower bound is available for $f$ in realistic problems (in the homogeneous case only).

The plan of the paper is as follows. In Section 2, we give a decomposition of $\bar{D}(f)$ in two terms, one of which includes cancellations, and the other is nonnegative. The former is shown to be controlled by $L^{1}$ type estimates in Section 3, and the latter is shown to give the desired estimate via the so-called Carleman representation in Section 4. Finally, in Section 5, we give some remarks about the role of intermediate collisions. 


\section{Splitting of $\bar{D}(f)$ and main result.}

Let us write

$$
\begin{aligned}
\sqrt{f^{\prime} f_{*}^{\prime}}-\sqrt{f f_{*}}= & \frac{1}{2}\left(\sqrt{f^{\prime}}-\sqrt{f}\right)\left(\sqrt{f_{*}^{\prime}}+\sqrt{f_{*}}\right) \\
& +\frac{1}{2}\left(\sqrt{f^{\prime}}+\sqrt{f}\right)\left(\sqrt{f_{*}^{\prime}}-\sqrt{f_{*}}\right) .
\end{aligned}
$$

Accordingly,

$$
\begin{aligned}
& \left(\sqrt{f^{\prime} f_{*}^{\prime}}-\sqrt{f f_{*}}\right)^{2} \\
& =\frac{1}{4}\left(\left(\sqrt{f^{\prime}}-\sqrt{f}\right)^{2}\left(\sqrt{f_{*}^{\prime}}+\sqrt{f_{*}}\right)^{2}\right. \\
& \left.\quad+\left(\sqrt{f^{\prime}}+\sqrt{f}\right)^{2}\left(\sqrt{f_{*}^{\prime}}-\sqrt{f_{*}}\right)^{2}\right) \\
& \quad+\frac{1}{2}\left(\sqrt{f^{\prime}}-\sqrt{f}\right)\left(\sqrt{f_{*}^{\prime}}+\sqrt{f_{*}}\right)\left(\sqrt{f^{\prime}}+\sqrt{f}\right)\left(\sqrt{f_{*}^{\prime}}-\sqrt{f_{*}}\right) .
\end{aligned}
$$

Reporting in (5) and using the classical change of variables $\left(v, v_{*}\right) \longrightarrow$ $\left(v^{\prime}, v_{*}^{\prime}\right)$, involutive and with unit Jacobian, we obtain

$$
\bar{D}(f)=S(f)+T(f),
$$

where

$$
\left\{\begin{array}{c}
S(f)=\frac{1}{2} \int d v d v_{*} d \omega B\left(v-v_{*}, \omega\right) \\
\cdot\left(\sqrt{f^{\prime}}-\sqrt{f}\right)^{2}\left(\sqrt{f_{*}^{\prime}}+\sqrt{f_{*}}\right)^{2}, \\
T(f)=\frac{1}{2} \int d v d v_{*} d \omega B\left(v-v_{*}, \omega\right)\left(f^{\prime}-f\right)\left(f_{*}^{\prime}-f_{*}\right) .
\end{array}\right.
$$

It is clear that in $T(f)$ one can expect strong cancellation effects, while $S(f)$ is nonnegative. We shall prove that $T(f)$ is well-defined without any regularity assumptions on $f$, while $S(f)$ is (locally) bounded below by a multiple of the square of some Sobolev norm of $\sqrt{f}$.

Before we state our results, let us discuss the assumptions for $B$. First of all, since $\bar{D}(f)$ is monotonic in $B$, it is sufficient, to obtain a general result, to treat the case when $B$ is "small". We shall therefore assume, without real loss of generality, 
Assumption A. $B\left(v-v_{*}, \omega\right)=\Phi\left(\left|v-v_{*}\right|\right) b(\cos \alpha)$, where

$$
\cos \alpha=k \cdot \omega, \quad k=\frac{v-v_{*}}{\left|v-v_{*}\right|} .
$$

Assumption B. $\Phi \in W^{1, \infty}\left(\mathbb{R}_{+}\right)$is a positive function with a bounded derivative, and is bounded from below uniformly, except maybe near 0 and $+\infty$.

The last assumption means of course that for all numbers $\varepsilon, R>0$ there exists $K(\varepsilon, R)>0$ such that $\Phi(|z|) \geq K(\varepsilon, R)>0$ if $\varepsilon \leq|z| \leq R$. This assumption is perfectly realistic from a physical point of view.

Now, we assume $b$ to be singular only for grazing collisions, in the sense

Assumption C. $b \in C([-1,1] \backslash\{0\})$, and

$$
b(\cos \alpha) \geq \frac{C}{|\cos \alpha|^{1+\nu}} \sim \frac{C}{\left(\frac{\pi}{2}-\alpha\right)^{1+\nu}}, \quad 0<\nu<2 .
$$

Here $\alpha \in[-\pi / 2, \pi / 2), C$ stands for arbitrary positive constants, and the sign $\sim$ only denotes similar asymptotic behaviour near the singularity $(\cos \alpha=0)$. Note in particular that $b$ is bounded below.

It is clear that the parameter $\nu$ measures the strength of the singularity of $B$ (note that if $\alpha \sim \pi / 2, d \omega$ and $d \alpha$ are roughly proportional). Let us comment on the assumption $\nu<2$. In order to do so, we introduce another (classical) representation for the collision operator (2), based on the unit vector $\sigma$ such that

$$
\left\{\begin{array}{l}
v^{\prime}=\frac{v+v_{*}}{2}+\frac{\left|v-v_{*}\right|}{2} \sigma, \\
v_{*}^{\prime}=\frac{v+v_{*}}{2}-\frac{\left|v-v_{*}\right|}{2} \sigma .
\end{array}\right.
$$

In this representation, Boltzmann's collision operator keeps the same form as in (2), except that $d \omega$ is changed into $d \sigma$, and $B\left(v-v_{*}, \omega\right)$ into

$$
\widetilde{B}\left(v-v_{*}, \sigma\right)=(2|k \cdot \omega|)^{-(N-2)} B\left(v-v_{*}, \omega\right) .
$$

Now, grazing collisions correspond to $k=\sigma$, i.e $k \cdot \sigma=1$. Assumptions $\mathrm{A}$ and $\mathrm{C}$ have to be replaced by their counterparts 
Assumption A'. $\widetilde{B}\left(v-v_{*}, \sigma\right)=\Phi\left(\left|v-v_{*}\right|\right) \widetilde{b}(k \cdot \sigma)$.

Assumption C'. $\widetilde{b} \in C([-1,1))$, and

(16) $\widetilde{b}(\cos \theta) \geq \frac{C}{\sin ^{N-2} \theta} \frac{1}{\theta^{1+\nu}} \sim \frac{C}{(\cos \theta-1)^{(N-1+\nu) / 2}}, \quad 0<\nu<2$.

Here $\theta \in[-\pi, \pi]$. Note that $\alpha=\pi / 2-\theta / 2$. Since $d \sigma$ is proportional to $\sin ^{N-2} \theta d \theta$, we see that

$$
\Lambda \equiv \int_{S^{N-1}} d \sigma \widetilde{b}(k \cdot \sigma)(1-k \cdot \sigma)=C_{N} \int_{0}^{\pi} d \theta \widetilde{b}(\cos \theta) \sin ^{2} \frac{\theta}{2}
$$

is finite if and only if $\nu<2$. Since $\Lambda$ has the physical meaning of a total cross-section for momentum transfer, we see that our assumption on $\nu$ is physically justified. This is consistent with the state of the art concerning the existence theory for the Boltzmann equation [24].

We can now state our main result. We use the classical notation

$\|f\|_{L_{1}^{1}}=\int_{\mathbb{R}^{N}}|f(v)|(1+|v|) d v, \quad L_{1}^{1}=\left\{f \in L^{1}\left(\mathbb{R}^{N}\right):\|f\|_{L_{1}^{1}}<\infty\right\}$.

Theorem 1. Let $f \in L_{1}^{1}\left(\mathbb{R}^{N}\right)$, and let $B$ be a cross-section satisfying assumptions $\mathrm{A}, \mathrm{B}, \mathrm{C}$. Then

i) There exists a constant $C$, independent of $f$, such that

$$
|T(f)| \leq C\|f\|_{L_{1}^{1}}\|f\|_{L^{1}}
$$

ii) Assume in addition that there exists a strictly positive function $\varepsilon(R)$ such that

$$
|v| \leq R \text { implies } f(v) \geq \varepsilon(R), \quad \text { for all } v \in \mathbb{R}^{N}, \text { for all } R>0 .
$$

Then there exists a strictly positive function $K(R)$, depending only on $\|f\|_{L_{1}^{1}}, \varepsilon(R)$ and the cross-section, such that

$$
S(f) \geq K(R)\|\sqrt{f}\|_{\dot{H}^{\nu / 2}(|v|<R)}^{2} .
$$

As an immediate consequence of the monotonicity of the entropy dissipation, we then deduce the 
Corollary 1.1. Let $f \in L_{1}^{1}\left(\mathbb{R}^{N}\right)$ such that $D(f)<\infty$, and $f$ satisfies the additional assumption of lower bound. Assume that $B \geq B_{0}$, where $B_{0}$ is a cross-section satisfying assumptions $\mathrm{A}, \mathrm{B}, \mathrm{C}$. Then $\sqrt{f} \in H_{\mathrm{loc}}^{\nu / 2}$.

REMARK. After completion of this work, we became aware of two Notes by Alexandre on the same subject [1], [2], where the Carleman representation is also used, but no splitting of the entropy dissipation as ours. It seems very difficult to understand whether the results therein are comparable to ours, but Alexandre kindly informed us that he had used this splitting independently in recent work, and obtained a bound very similar to ours, as well as related results in the (very difficult) inhomogeneous case. The proofs by Alexandre rely on the theory of pseudo-differential operators. Desvillettes has also shown us some of his partial results in collaboration with Wennberg, which are consistent with both our conclusions and our method of proof, but do not start from the entropy estimate.

\section{Cancellation effects for grazing collisions.}

In this section, we prove the estimate (17). First, by the usual change of variables $\left(v, v_{*}\right) \longrightarrow\left(v^{\prime}, v_{*}^{\prime}\right)$,

$$
T(f)=\int d v d v_{*} d \omega B\left(v-v_{*}, \omega\right) f\left(f_{*}-f_{*}^{\prime}\right)=\int_{\mathbb{R}^{N}} d v f(v) G(v)
$$

with

$$
G(v)=\int_{\mathbb{R}^{N} \times S^{N-1}} d v_{*} d \omega B\left(v-v_{*}, \omega\right)\left(f_{*}-f_{*}^{\prime}\right) .
$$

We now proceed to estimate $G$. We turn to the $\sigma$-representation (15) and use Assumption A:

$$
G(v)=\int_{\mathbb{R}^{N} \times S^{N-1}} d v_{*} d \sigma \Phi\left(\left|v-v_{*}\right|\right) \widetilde{b}(k \cdot \sigma)\left(f_{*}-f_{*}^{\prime}\right) .
$$

Now, for fixed $\sigma$, the change of variables $v_{*} \longrightarrow v_{*}^{\prime}$ is valid, and an easy computation yields

$$
d v_{*}^{\prime}=\frac{1}{2^{N}}(1+(k \cdot \sigma)) d v_{*}=\frac{1}{2^{N}}(1+\cos \theta) d v_{*} .
$$


Let $k^{\prime}=\left(v-v_{*}^{\prime}\right) /\left|v-v_{*}^{\prime}\right|$. An elementary geometric argument shows that

$$
1+(k \cdot \sigma)=1+\cos \theta=2 \cos ^{2} \frac{\theta}{2}=2\left(k^{\prime} \cdot \sigma\right)^{2} .
$$

By symmetrization, one can assume that $b(\cos \theta)$ is supported in $(-\pi / 2$ $\leq \theta \leq \pi / 2)$, i.e. $(k \cdot \sigma) \geq 0$ (this can be seen as a consequence of the indiscernability of the particles), so that the Jacobian in (19) is bounded below.

For given $\sigma$, let us introduce

$$
\psi_{\sigma}: v_{*}^{\prime} \longmapsto v_{*}
$$

It is easy to check that for given $\left(v, v_{*}^{\prime}, \sigma\right)$ such that $\left(v-v_{*}^{\prime}, \sigma\right)>0$, the equation with unknown $v_{*} \in \mathbb{R}^{N}$

$$
\psi_{\sigma}\left(v_{*}^{\prime}\right)=v_{*}
$$

is uniquely solvable (note that if $v_{*}^{\prime}$ is given by (15), then $2\left(v-v_{*}^{\prime}, \sigma\right)=$ $\left.\left(v-v_{*}, \sigma\right)+\left|v-v_{*}\right|>0\right)$. Moreover, the condition $\left(v-v_{*}, \sigma\right)>0$ is equivalent to

$$
\left(k^{\prime}, \sigma\right)=\left(\frac{v-v_{*}^{\prime}}{\left|v-v_{*}^{\prime}\right|}, \sigma\right)>\frac{1}{\sqrt{2}} .
$$

See the geometric interpretation of $\psi_{\sigma}$ in Figure 1.

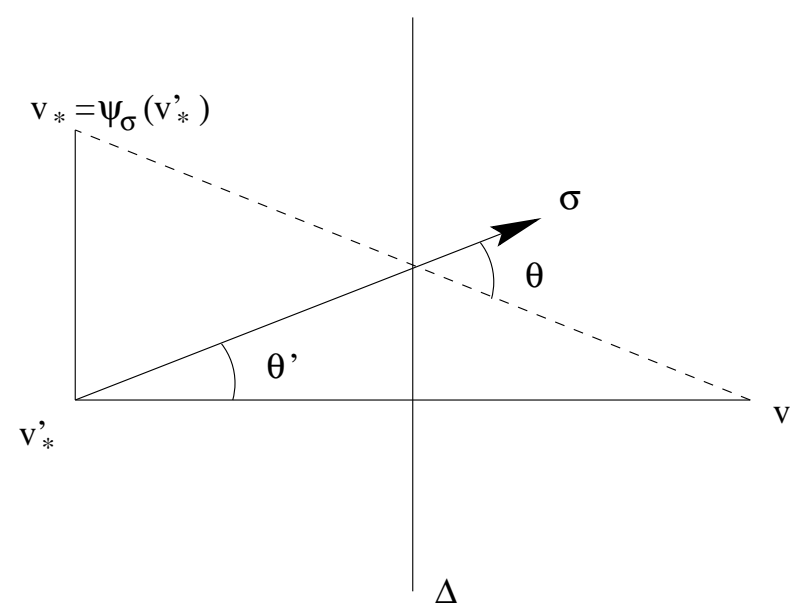

Figure 1. $\Delta$ is the mediatrix of $\left(v, v_{*}^{\prime}\right) ; \cos \theta=k \cdot \sigma ; \cos \theta^{\prime}=\cos (\theta / 2)$. 
Accordingly, we write (using (19) and (20))

$$
\begin{gathered}
G(v)=\int_{k \cdot \sigma>0} d v_{*} d \sigma \Phi\left(\left|v-v_{*}\right|\right) \widetilde{b}(k \cdot \sigma) f\left(v_{*}\right) \\
-\int_{k^{\prime} \cdot \sigma>1 / \sqrt{2}} d v_{*}^{\prime} d \sigma \frac{2^{N-1}}{\left(k^{\prime} \cdot \sigma\right)^{2}} \Phi\left(\left|v-\psi_{\sigma}\left(v_{*}^{\prime}\right)\right|\right) \\
\cdot \widetilde{b}\left(2\left(k^{\prime} \cdot \sigma\right)^{2}-1\right) f\left(v_{*}^{\prime}\right) .
\end{gathered}
$$

Since the integration variable in the second integral is a dummy one, we conclude that

$$
G(v)=\int_{\mathbb{R}^{N}} d v_{*} f\left(v_{*}\right) C\left(v, v_{*}\right)
$$

with

$C\left(v, v_{*}\right)=\int_{S^{N-1}} d \sigma\left(\Phi\left(\left|v-v_{*}\right|\right) \tilde{b}(k \cdot \sigma) \mathbf{1}_{\{k \cdot \sigma>0\}}\right.$

$$
\begin{gathered}
\left.-\Phi\left(\left|v-\psi_{\sigma}\left(v_{*}\right)\right|\right) \frac{2^{N-1}}{(k \cdot \sigma)^{2}} \widetilde{b}\left(2(k \cdot \sigma)^{2}-1\right) 1_{\{k \cdot \sigma>1 / \sqrt{2}\}}\right) \\
=\Phi\left(\left|v-v_{*}\right|\right) \int_{S^{N-1}} d \sigma \widetilde{b}(k \cdot \sigma) \mathbf{1}_{\{0<k \cdot \sigma \leq 1 / \sqrt{2}\}} \\
+\Phi\left(\left|v-v_{*}\right|\right) \int_{S^{N-1}} d \sigma\left(\widetilde{b}(k \cdot \sigma)-\frac{2^{N-1}}{(k \cdot \sigma)^{2}} \widetilde{b}\left(2(k \cdot \sigma)^{2}-1\right)\right) \\
+\int_{S^{N-1}} d \sigma\left(\Phi\left(\left|v-v_{*}\right|\right)-\Phi\left(\left|v-\psi_{\sigma}\left(v_{*}\right)\right|\right)\right) \\
\cdot \frac{2^{N-1}}{(k \cdot \sigma)^{2}} \widetilde{b}\left(2(k \cdot \sigma)^{2}-1\right) \mathbf{1}_{\{k \cdot \sigma>1 / \sqrt{2}\}} .
\end{gathered}
$$

As an immediate consequence of Assumptions B and C, the expression (23) is bounded by a constant, independently of $v, v_{*}$. Let us now consider the last term (25). Still using the notation $\cos \theta=k \cdot \sigma$, we see that

$$
\left|v-\psi_{\sigma}\left(v_{*}\right)\right|=\frac{\left|v-v_{*}\right|}{\cos \theta}
$$

so that

$$
\left|\Phi\left(\left|v-v_{*}\right|\right)-\Phi\left(\left|v-\psi_{\sigma}\left(v_{*}\right)\right|\right)\right| \leq\left\|\Phi^{\prime}\right\|_{\infty}\left|v-v_{*}\right|\left(\frac{1}{\cos \theta}-1\right) .
$$


Therefore, as a consequence of Assumption C, the integral (25) is bounded by

$$
\begin{aligned}
C_{N}\left\|\Phi^{\prime}\right\|_{\infty} \mid v & -v_{*} \mid \int_{0}^{\pi / 4} d \theta \widetilde{b}(\cos 2 \theta)(\cos \theta-1) \sin ^{N-2} \theta \\
\leq & C_{N}\left\|\Phi^{\prime}\right\|_{\infty}\left(|v|+\left|v_{*}\right|\right) \int_{0}^{\pi / 4} d \theta \theta^{1-\nu} \\
\leq & C\left(|v|+\left|v_{*}\right|\right)
\end{aligned}
$$

for some $\nu<2$, where the constant $C$ depends only on $N$ and the constants in Assumptions B, C.

Finally, we estimate the integral in (24). By a spherical change of variables, this is

$$
\begin{aligned}
& C_{N} \int_{0}^{\pi / 4} d \theta \sin ^{N-2} \theta\left(\widetilde{b}(\cos \theta)-\frac{2^{N-1}}{\cos ^{2} \theta} \widetilde{b}(\cos 2 \theta)\right) \\
&=C_{N} \int_{0}^{\pi / 4} d \theta \sin ^{N-2} \theta \widetilde{b}(\cos \theta) \\
&-C_{N} \int_{0}^{\pi / 2} d \theta \sin ^{N-2}\left(\frac{\theta}{2}\right) \frac{2^{N-2}}{\cos ^{2}\left(\frac{\theta}{2}\right)} \widetilde{b}(\cos \theta) .
\end{aligned}
$$

By the formula $\sin ^{N-2}(\theta / 2) 2^{N-2} \cos ^{N-2}(\theta / 2)=\sin ^{N-2} \theta$, we get

$$
\begin{aligned}
C_{N} \int_{0}^{\pi / 4} d \theta \sin ^{N-2} \theta \widetilde{b}(\cos \theta)\left(1-\frac{1}{\cos ^{N}\left(\frac{\theta}{2}\right)}\right) \\
-C_{N} \int_{\pi / 4}^{\pi / 2} d \theta \sin ^{N-2}\left(\frac{\theta}{2}\right) \frac{2^{N-2}}{\cos ^{2}\left(\frac{\theta}{2}\right)} \widetilde{b}(\cos \theta) .
\end{aligned}
$$

The second integral is convergent by Assumption C, and so is also the first, since for $N \geq 2$,

$$
1-\frac{1}{\cos ^{N}\left(\frac{\theta}{2}\right)}=O\left(\theta^{2}\right), \quad \text { as } \theta \longrightarrow 0 .
$$

In the end, we find

$$
\left|C\left(v, v_{*}\right)\right| \leq C\left(|v|+\left|v_{*}\right|\right),
$$


whence the conclusion.

\section{The Carleman representation.}

We now transform $S(f)$ into an expression looking like the square of a (fractional) Sobolev norm. To this purpose, we use the so-called Carleman representation, which was actually introduced by Carleman in [6], and later reformulated by Wennberg [26]. It should be noted that the purpose of Wennberg is also to obtain regularity estimates, though in a very different context.

The idea of the Carleman representation is to replace the set of variables $\left(v, v_{*}, \omega\right)$ by the set $\left(v, v^{\prime}, v_{*}^{\prime}\right)$, where $v \in \mathbb{R}^{N}, v^{\prime} \in \mathbb{R}^{N}$ and $v_{*}^{\prime} \in E_{v v^{\prime}}$, the hyperplane going through $v$ and orthogonal to $v-v^{\prime}$.

Let us recall briefly the argument. Following Wennberg, we introduce the variable $q=\left|v-v^{\prime}\right|$ and note that

$$
\left\{\begin{array}{l}
v^{\prime}=v+q \omega, \\
v_{*}=v_{*}^{\prime}+q \omega .
\end{array}\right.
$$

Since $\omega$ and $E_{v v^{\prime}}$ are orthogonal, the second relation entails $d v_{*}=$ $d v_{*}^{\prime} d q$, where $d v_{*}^{\prime}$ denote the Lebesgue measure on $E_{v v^{\prime}}$. On the other hand, $d v^{\prime}=q^{N-1} d q d \omega$. Hence,

$$
d v_{*} d \omega=d v_{*}^{\prime} d q d \omega=\frac{d v_{*}^{\prime} d v^{\prime}}{q^{N-1}}=\frac{d v_{*}^{\prime} d v^{\prime}}{\left|v-v^{\prime}\right|^{N-1}} .
$$

Note that $d v_{*}^{\prime}$ is a $(N-1)$-dimensional measure, while $d v^{\prime}$ is $N$-dimensional.

By Assumption A, and since $\cos \alpha=\left|v-v^{\prime}\right| /\left|v-v_{*}\right|,\left|v-v_{*}\right|=$ $\left|v^{\prime}-v_{*}^{\prime}\right|$, we obtain

$$
\begin{aligned}
S(f)=\frac{1}{2} \int_{\mathbb{R}^{2 N}} \frac{d v d v^{\prime}}{\left|v-v^{\prime}\right|^{N-1}} \\
\cdot\left(\int_{E_{v v^{\prime}}} d v_{*}^{\prime} \Phi\left(\left|v^{\prime}-v_{*}^{\prime}\right|\right)\left(\sqrt{f_{*}^{\prime}}+\sqrt{f_{*}}\right)^{2} b\left(\frac{\left|v^{\prime}-v\right|}{\left|v^{\prime}-v_{*}^{\prime}\right|}\right)\right) \\
\cdot\left(\sqrt{f^{\prime}}-\sqrt{f}\right)^{2} .
\end{aligned}
$$

Note that (for given $\left.v, v^{\prime}\right) v_{*}^{\prime}$ and $v_{*}$ describe parallel planes (in fact, $\left.v_{*}=\left(v^{\prime}-v\right)+v_{*}^{\prime}\right)$. 
Using Assumption C, for some positive constant $K$,

$$
b\left(\frac{\left|v^{\prime}-v\right|}{\left|v^{\prime}-v_{*}^{\prime}\right|}\right) \geq K \frac{\left|v^{\prime}-v_{*}^{\prime}\right|^{1+\nu}}{\left|v^{\prime}-v\right|^{1+\nu}} .
$$

Thus, we can write

$$
S(f) \geq K \int_{\mathbb{R}^{2 N}} d v d v^{\prime} A\left(v, v^{\prime}\right) \frac{\left(\sqrt{f\left(v^{\prime}\right)}-\sqrt{f(v)}\right)^{2}}{\left|v^{\prime}-v\right|^{N+\nu}}
$$

with

$$
A\left(v, v^{\prime}\right)=\int_{E_{v v^{\prime}}} d v_{*}^{\prime} \Phi\left(\left|v^{\prime}-v_{*}^{\prime}\right|\right)\left(f_{*}^{\prime}+f_{*}\right)\left|v^{\prime}-v_{*}^{\prime}\right|^{1+\nu} .
$$

Let us set $\Psi(|z|)=|z|^{1+\nu} \Phi(|z|)$. By Assumption B, $\Psi$ can vanish only near 0 and $\infty$. We note that

$$
A\left(v, v^{\prime}\right)=\int_{E_{v v^{\prime}}} d v_{*}^{\prime} \Psi\left(\left|v^{\prime}-v_{*}^{\prime}\right|\right) f\left(v_{*}^{\prime}\right)+\int_{E_{v^{\prime} v}} d v_{*} \Psi\left(\left|v-v_{*}\right|\right) f\left(v_{*}\right) .
$$

The estimate (29) is a Sobolev estimate as soon as $A$ is bounded from below. This is clearly true locally if $f$ is locally bounded from below (it suffices in fact that all integrals of $f$ upon bounded portions of hyperplanes going through $v$ be bounded from below, locally in $v$ ). This completes our proof.

REMARK. The coefficients $A\left(v, v^{\prime}\right)$ are given by Radon transforms, and therefore are likely to be smooth, in some sense; this remark, combined with the method of the next section, could help relax the assumption of local lower bound.

\section{The role of intermediate collisions.}

In this section, we only want to emphasize how the method applied above can be refined by the use of intermediate collisions. Indeed, the coefficients $A\left(v, v^{\prime}\right)$ of the previous section measure, in some sense, the number of collisions in which the particles change their velocity from $v$ to $v^{\prime}$. The "gain of regularity" therefore depends upon these coefficients. But particles can also gain an arbitrary velocity $v^{\prime \prime} \in \mathbb{R}^{N}$ before they gain the velocity $v^{\prime}$. We shall see how to make this vague physical idea 
more precise. Even though we did not find any application for it, we think it likely that this method could be useful for related problems.

Let us rewrite the estimate (29) with $v^{\prime}$ replaced by $w$ : up to a constant,

$$
\int_{\mathbb{R}^{2 N}} d v d w A(v, w) \frac{(\sqrt{f(v)}-\sqrt{f(w)})^{2}}{|v-w|^{N+\nu}} \leq S(f) .
$$

As a consequence, for any $C, \varepsilon>0$,

$$
\begin{aligned}
\int_{\mathbb{R}^{3 N}} d v d w d v^{\prime} A(v, w) \mathbf{1}_{\left\{|v-w| \leq C\left|v-v^{\prime}\right| \leq C\right\}} & \frac{(\sqrt{f(v)}-\sqrt{f(w)})^{2}}{\left|v-v^{\prime}\right|^{2 N+\nu-\varepsilon}} \\
& \leq C(S(f), C, \varepsilon) \\
& <\infty .
\end{aligned}
$$

Indeed, in the domain of integration, $\left|v^{\prime}-w\right| \leq(1+C)$, so that the integral (30) is bounded by

$$
\begin{aligned}
C^{N+\nu}\left(\int_{\mathbb{R}^{2 N}} d v d w A(v, w) \frac{(\sqrt{f(v)}-\sqrt{f(w)})^{2}}{|v-w|^{N+\nu}}\right) \\
\cdot\left(\int_{\left|v^{\prime}-w\right| \leq 1+C} d v^{\prime} \frac{1}{\left|v^{\prime}-w\right|^{N-\varepsilon}}\right) .
\end{aligned}
$$

Similarly,

$$
\begin{gathered}
\int_{\mathbb{R}^{3 N}} d v d v^{\prime} d w A\left(w, v^{\prime}\right) \mathbf{1}_{\left\{\left|w-v^{\prime}\right| \leq C\left|v-v^{\prime}\right| \leq C\right\}} \\
\cdot \frac{\left(\sqrt{f(w)}-\sqrt{f\left(v^{\prime}\right)}\right)^{2}}{\left|v-v^{\prime}\right|^{2 N+\nu-\varepsilon}}<\infty .
\end{gathered}
$$

Since

$$
\left(\sqrt{f(v)}-\sqrt{f\left(v^{\prime}\right)}\right)^{2} \leq 2(\sqrt{f(v)}-\sqrt{f(w)})^{2}+2\left(\sqrt{f(w)}-\sqrt{f\left(v^{\prime}\right)}\right)^{2},
$$

we get by adding up (30) and (31)

$$
\begin{aligned}
\int_{\mathbb{R}^{2 N}} d v d v^{\prime} \mathbf{1}_{\left\{\left|v-v^{\prime}\right| \leq 1\right\}} & \left.d w \min \left\{A(v, w), A\left(w, v^{\prime}\right)\right\}\right) \\
\cdot & \left(\int_{|v-w|,\left|v^{\prime}-w\right| \leq C\left|v-v^{\prime}\right|} d w\right. \\
& \cdot \frac{\left(\sqrt{f(v)}-\sqrt{f\left(v^{\prime}\right)}\right)^{2}}{\left|v-v^{\prime}\right|^{2 N+\nu-\varepsilon}}<\infty .
\end{aligned}
$$


Since, of course,

$$
\int_{\mathbb{R}^{2 N}} d v d v^{\prime} \mathbf{1}_{\left\{\left|v-v^{\prime}\right| \geq 1\right\}} \frac{\left(\sqrt{f(v)}-\sqrt{f\left(v^{\prime}\right)}\right)^{2}}{\left|v-v^{\prime}\right|^{N+\nu}} \leq C\|f\|_{L^{1}}<\infty,
$$

we see that, up to an arbitrarily small degradation in the Sobolev exponent, the coefficients $A\left(v, v^{\prime}\right)$ can be replaced by

$$
\bar{A}\left(v, v^{\prime}\right)
$$

$$
\equiv \frac{1}{\left|v-v^{\prime}\right|^{N}} \int_{\max \left\{|v-w|,\left|v^{\prime}-w\right|\right\} \leq C\left|v-v^{\prime}\right|} d w \min \left\{A(v, w), A\left(w, v^{\prime}\right)\right\} .
$$

Since the volume of

$$
\left\{w \in \mathbb{R}^{N}: \max \left\{|v-w|,\left|v^{\prime}-w\right|\right\} \leq C\left|v-v^{\prime}\right|\right\}
$$

behaves like $\left|v-v^{\prime}\right|^{N}$, we see that $\bar{A}$ is a kind of average of $A$, and hence more likely to be bounded from below than $A$. Of course the procedure can be iterated as many times as desired. We did not go further in this investigation.

Acknowledgement. We thank P.-L. Lions for showing us his Note [19] and discussing the results therein.

\section{References.}

[1] Alexandre, R., Sur l'opérateur de Boltzmann non linéaire 3D sans troncature angulaire. C. R. Acad. Sci. Paris 326 (1998), 165-168.

[2] Alexandre, R., Sur le taux de dissipation d'entropie sans troncature angulaire. C. R. Acad. Sci. Paris 326 (1998), 311-315.

[3] Arkeryd, L., Intermolecular forces of infinite range and the Boltzmann equation. Arch. Rational Mech. Anal. 77 (1981), 11-21.

[4] Arsen'ev, A. A., Buryak, O. E., On the connection between a solution of the Boltzmann equation and a solution of the Landau-Fokker-Planck equation. Math. USSR Sbornik 69 (1991), 465-478.

[5] Bouchut, F., Desvillettes, L., A proof of the smoothing properties of the positive parts of Boltzmann's kernel. Revista Mat. Iberoamericana 14 (1998), 47-61. 
[6] Carleman, T., Problèmes Mathématiques dans la Théorie Cinétique des Gaz. Almqvist \& Wiksell, 1957.

[7] Carlen, E., Carvalho, M., Strict entropy production bounds and stability of the rate of convergence to equilibrium for the Boltzmann equation. J. Stat. Phys. 67 (1992), 575-608.

[8] Carlen, E., Desvillettes, L., Work in progress (personal communication).

[9] Cercignani, C., A remarkable estimate for the solutions of the Boltzmann equation. Appl. Math. Lett. 5 (1992), 59-62.

[10] Desvillettes, L., About the regularizing properties of the non-cut-off Kac equation. Comm. Math. Phys. 168 (1995), 417-440.

[11] Desvillettes, L., Regularization for the non-cutoff 2D radially symmetric Boltzmann equation with a velocity dependent cross section. Transp. Theory Stat. Phys. 25 (1996), 383-394.

[12] Desvillettes, L., Regularization properties of the 2-dimensional non radially symmetric non cutoff spatially homogeneous Boltzmann equation for Maxwellian molecules. Transp. Theory Stat. Phys. 26 (1997), 341357.

[13] Desvillettes, L., Villani, C., On the spatially homogeneous Landau equation for hard potentials. Part I: Existence, uniqueness and smoothness. To appear in Comm. Partial Diff. Equations.

[14] Desvillettes, L., Villani, C., On the spatially homogeneous Landau equation for hard potentials. Part II: $H$-theorem and applications. To appear in Comm. Partial Diff. Equations.

[15] Goudon, T., On the Boltzmann equation and its relations to the LandauFokker-Planck equation: influence of grazing collisions. C. R. Acad. Sci. Paris 324 (1997), 265-270.

[16] McKean. H. P. Jr., Speed of approach to equilibrium for Kac's caricature of a Maxwellian gas. Arch. Rational Mech. Anal. 21 (1966), 343-367.

[17] Lions, P. L., Compactness in Boltzmann's equation via Fourier integral operators and applications. J. Math. Kyoto Univ. 34 (1994), 391-427.

[18] Lions, P. L., On Boltzmann and Landau equations. Phil. Trans. R. Soc. Lond. 346 (1994), 191-204.

[19] Lions, P. L., Regularity and compactness for Boltzmann collision operators without angular cut-off. C. R. Acad. Sci. Paris 326 (1998), $37-41$.

[20] Maxwell, J. C., On the dynamical theory of gases. Phil. Trans. R. Soc. Lond. 157 (1867), 49-88.

[21] Proutière, A., New results of regularization for weak solutions of Boltzmann equation. Preprint, 1996. 
[22] Pulvirenti, A., Wennberg, B., A Maxwellian lower bound for solutions to the Boltzmann equation. Comm. Math. Phys. 183 (1997), 145-160.

[23] Toscani, G., New a priori estimates for the spatially homogeneous Boltzmann equation. Cont. Mech. Thermodyn. 4 (1992), 81-93.

[24] Villani, C., On a new class of weak solutions to the spatially homogeneous Boltzmann and Landau equations. Arch. Rational Mech. Anal. 143 (1998), 273-307.

[25] Villani, C., Fisher information bounds for the Boltzmann equation. $J$. Math. Pures et Appl. 77 (1998), 821-837.

[26] Wennberg, B., Regularity in the Boltzmann equation and the Radon transform. Comm. Partial Diff. Equations 19 (1994), 2057-2074.

Recibido: 1 de junio de 1.998

Cédric Villani

École Normale Supérieure, DMI

45 rue d'Ulm

75230 Paris Cedex 05, FRANCE

villani@dmi .ens.fr 